\section{Implantação do protocolo de vigilância do óbito com menção de tuberculose e seus efeitos na vigilância de um município brasileiro de grande porte}

\author{
Implementation of a protocol for surveillance \\ of deaths with mention of tuberculosis and the \\ effects on surveillance in a large Brazilian city
}

Implantación del protocolo de vigilancia de óbitos con mención de tuberculosis y sus efectos en la vigilancia de un municipio brasileño de gran tamaño
Dinah Carvalho Cordeiro 1,2

Maria Jacirema Ferreira Gonçalves 1

\section{Resumo}

O objetivo deste estudo foi avaliar o grau de implantação e de impacto do protocolo de vigilância do óbito por tuberculose (TB) em um município de grande porte e com elevada carga de TB. Para esta pesquisa avaliativa, elaborou-se um modelo lógico e matriz de julgamento. Os dados primários foram coletados a partir de um questionário aplicado aos profissionais e os dados secundários foram obtidos na Secretaria Municipal de Saúde de Manaus, Amazonas, Brasil. O impacto da adoção do protocolo foi analisado pelos indicadores de resultados e a evolução das taxas de incidência e mortalidade por TB, a partir da regressão linear simples e variação percentual. O grau de implantação da vigilância do óbito com menção de TB identificado foi parcial (51\%). Quanto à evolução dos indicadores de incidência e mortalidade por TB, observou-se tendência significativa de aumento da taxa da incidência de TB no período de 2006 a 2019. A mortalidade por TB e a proporção dos óbitos com confirmação laboratorial para TB apresentaram incremento após a implantação do protocolo, indicando aumento da vigilância. A implantação parcial da vigilância do óbito de TB indica necessidades de mudanças no processo de trabalho da equipe que gerencia o Sistema de Informações sobre Mortalidade e o Programa de Controle da TB municipal. As mudanças observadas demonstram mais qualificação das informações do óbito por TB, assim como a vigilância no diagnóstico da TB contribuiu para a definição de casos com confirmação laboratorial para $T B$.

Tuberculose; Mortalidade; Avaliação em Saúde; Vigilância Epidemiológica

\author{
Correspondência \\ D. C. Cordeiro \\ Av. Prof. Nilton Lins 877, Manaus, AM 69058-030, Brasil. \\ dinah.cordeiro@pmm.am.gov.br \\ 1 Universidade Federal do Amazonas, Manaus, Brasil. \\ 2 Secretaria Municipal de Saúde de Manaus, Manaus, Brasil.
}




\section{Introdução}

Apesar de todos os esforços para controlar a tuberculose (TB), no Brasil, em 2019, foram notificados 73.864 casos novos de TB e 4.490 óbitos pela doença. Entre os estados brasileiros, o Amazonas apresentou a maior taxa de incidência do país com 75,4 casos/100 mil habitantes, e, no âmbito das capitais, Manaus (Amazonas) destacou-se entre as maiores taxas de incidência da doença (104,6 casos/100 mil habitantes) e de mortalidade por TB (4,5 óbitos/100 mil habitantes) 1.

O óbito por TB é considerado um evento evitável e importante problema de saúde pública, sua ocorrência pode revelar dificuldade de acesso ao diagnóstico e tratamento da TB 2,3. Além disso, estudos prévios estimam que haja subnotificação de casos e fragilidades nos sistemas de informação e vigilância da $\mathrm{TB}$, refletindo um cenário epidemiológico irreal 4,5 . Na vigilância da $\mathrm{TB}$, os dados gerados pelos sistemas de informação subsidiam o processo de planejamento, monitoramento, avaliação e tomada de decisão, sendo fundamental uma rotina de verificação e correção das inconsistências pelos programas de controle da TB (PCT) municipais e estaduais 6.

Apesar das recomendações de verificação e correção das inconsistências, a qualidade dos dados nos sistemas de informação parecem ser nós críticos para vigilância da TB em todo o Brasil 4,5,7,8,9. Como estratégia para corrigir qualiquantitativamente os sistemas de informação, compreender as circunstâncias que levaram ao óbito e qualificar a assistência à saúde, o Ministério da Saúde, por meio do Departamento de Doenças de Condições Crônicas e Infecções Sexualmente Transmissíveis (DCCI), publicou em 2017 o Protocolo de Vigilância do Óbito com Menção de Tuberculose nas Causas de Morte 3.

A recomendação do Programa Nacional de Controle da Tuberculose (PNCT), no Brasil, é que o óbito com menção de TB nas causas de morte seja identificado e investigado, seguindo as diretrizes e metas para vigilância do óbito de TB. Após as investigações, a orientação é que um grupo de trabalho multiprofissional seja formado para discussão acerca da ocorrência do óbito com menção de TB, identificando as circunstâncias para ocorrência do óbito e as fragilidades nos diversos níveis de atenção à saúde, além de propor recomendações para qualificar os sistemas de informação e a assistência à saúde do paciente com TB 3,6.

Quando uma intervenção nova é implementada, é pertinente realizar a análise de implantação 10, pois permitirá identificar a dinâmica de implantação e os fatores explicativos críticos. Dentre as várias abordagens adotadas na avaliação em saúde está o modelo de Donabedian ${ }^{11}$, que estabelece a tríade "estrutura - processo - resultado", e esse modelo julgou-se adequado a esta proposta de análise, pois dispõe de atributos (eficácia, efetividade, eficiência, otimização, aceitabilidade, legitimidade e equidade) que traduzem a qualidade nos serviços e se sustenta em três componentes da avaliação 11,12 .

No âmbito das publicações científicas, há estudos sobre os aspectos epidemiológicos, socioeconômicos e demográficos dos óbitos por TB 13,14,15,16, assim como a verificação da qualidade dos sistemas de informação para vigilância da doença 5,7,17. Entretanto ainda não foram identificados estudos relacionados à avaliação de implantação do protocolo de vigilância do óbito com menção de TB, considerando ser estratégia recente no Brasil.

O objetivo deste estudo foi avaliar o grau de implantação e de impacto da adoção do protocolo de vigilância do óbito por TB em um município de grande porte e com elevada carga de TB.

\section{Métodos}

Esta é uma pesquisa avaliativa do tipo análise de implantação e de impacto da vigilância do óbito com menção de TB nas causas de morte, tendo como estratégia metodológica estudo de caso único, por meio de modelo lógico e matriz de julgamento.

A pesquisa foi aplicada no PCT do Município de Manaus. A cidade está situada na Região Norte do Brasil, com população estimada em 2020 de 2.219 .580 habitantes, que representa 52,7\% da população do Estado do Amazonas 18. A cobertura da Estratégia Saúde da Família em 2020 variou de 39,36\% a $45,52 \%$, e a da atenção primária à saúde variou de $51,37 \%$ a $66,85 \% 19$.

Historicamente, Manaus destaca-se por estar entre as capitais com as maiores taxas de incidência da TB no país e por ser a quinta capital com maior taxa de mortalidade pela doença em 2018 1. As ações de vigilância em saúde são desenvolvidas pelos Departamentos de Vigilância Ambiental e Epi- 
demiológica e pelos Distritos de Saúde que compõem a estrutura organizacional da Secretaria Municipal de Saúde de Manaus (Semsa). Para efeito de planejamento, desconcentração urbana, agilidade administrativa e descentralização das ações de saúde, o território de Manaus está dividido em cinco Distritos Sanitários (DS): Leste, Norte, Oeste, Sul e Rural 20.

O município foi sede para realização do piloto de implantação da vigilância do óbito de TB em 2017. Na ocasião, foram discutidos e elaborados os fluxos e as fichas de investigação ${ }^{3}$. Desde então, a equipe técnica do PCT de Manaus, em parceria com diversos setores da saúde, tem investigado óbitos com menção da TB ocorridos em seu território. Logo, após três anos, é pertinente avaliar a implantação e o impacto da vigilância do óbito com menção de TB, pois seus resultados têm potencial de redirecionar os caminhos dessa implantação e auxiliar na implementação da vigilância do óbito em outros cenários.

Os dados primários foram coletados a partir de um questionário autoaplicável, de autoria própria, contendo questões voltadas ao preenchimento da matriz de julgamento (indicadores de estrutura, processo e resultado por componente do modelo lógico), formulado a partir das referências bibliográficas 3,6. As fontes de informação dos dados secundários obtidos na Semsa - Manaus foram: as notificações de TB do Sistema de Informação de Agravos de Notificação (SINAN) e os óbitos com menção de TB na causa básica do Sistema de Informações sobre Mortalidade (SIM), conforme os códigos A15 ao A19 da 10a revisão da Classificação Internacional de Doenças (CID-10). O período de 2005 a 2019 foi selecionado para análise, quando as ações de controle da TB já estavam descentralizadas para a atenção primária à saúde de Manaus 21, e um intervalo maior pode evidenciar alterações epidemiológicas da doença, incluindo o impacto da implantação da vigilância de óbitos por TB, que ocorreu em 2017. Os dados da população estimada e projeção de residentes em Manaus no período de 2005 a 2019 e do Censo Demográfico de 2010 foram obtidos no portal do Instituto Brasileiro de Geografia e Estatística 18, os quais compuseram os denominadores das taxas calculadas.

O critério de inclusão dos participantes era atuação técnica mediante o PCT em nível central e distrital, com desenvolvimento nas atividades de vigilância do óbito com menção de TB e atuação na gestão do SIM.

Para a coleta de dados, foi construído um modelo lógico de avaliação da vigilância do óbito com menção de TB nas causas de morte (Figura 1), adaptado do modelo lógico da vigilância do óbito infantil 22, com base em documentos oficiais do PCT que tratam da vigilância do óbito com TB 3,6.

O modelo lógico (Figura 1) contém quatro colunas: na primeira coluna estão os componentes que norteiam a vigilância do óbito com menção de TB - identificação do óbito, investigação epidemiológica, discussão dos óbitos, encaminhamento de propostas e qualificação dos sistemas de informação; na segunda coluna constam os insumos essenciais para a operacionalização das ações; na terceira estão as atividades para execução dos componentes; e, na quarta, os resultados, metas e efeitos esperados.

Posteriormente, foi elaborada, heuristicamente, uma matriz de indicadores e julgamento por componente do modelo lógico relacionados à estrutura, ao processo e ao resultado 3,11,22. Para cada indicador, foram estabelecidos parâmetros de acordo com a recomendação do PNCT e, para os indicadores sem parâmetros, utilizou-se o critério da razoabilidade, considerando o que seria ideal para o bom funcionamento do PCT (Quadro 1).

Para análise dos dados, foram elaborados 56 indicadores para os quatro componentes do modelo lógico, sendo: 48 relacionados ao grau de implantação (20 de estrutura e 28 de processo) e 8 de resultados. O julgamento dos indicadores foi por meio de pontuações de 0 a 5 pontos 22,23. Para alguns indicadores cuja fonte de informações foi composta pelos diversos profissionais, os valores foram somados e calculada a média.

Por componente, os pontos foram distribuídos da seguinte forma: (a) identificação dos óbitos - 10 pontos; (b) investigação epidemiológica - 40 pontos; (c) discussão dos óbitos - 30 pontos; e (d) encaminhamento de proposta e qualificação dos sistemas de informação - 20 pontos. $\mathrm{O}$ valor máximo possível em toda a matriz foi 100 pontos, em caso de desempenho plenamente satisfatório. Os resultados foram apresentados em número absoluto e percentual ( $\Sigma$ de pontos obtidos/pontos máximos*100).

Foram atribuídas as menores pontuações aos componentes "identificação do óbito" e "encaminhamento de propostas e qualificação dos sistemas de informações"; a primeira por ser uma atividade realizada em parceria com a equipe responsável pela gestão do SIM, e a segunda por ser um desdo- 
Figura 1

Modelo lógico de avaliação da vigilância do óbito com menção de tuberculose (TB) nas causas de morte.

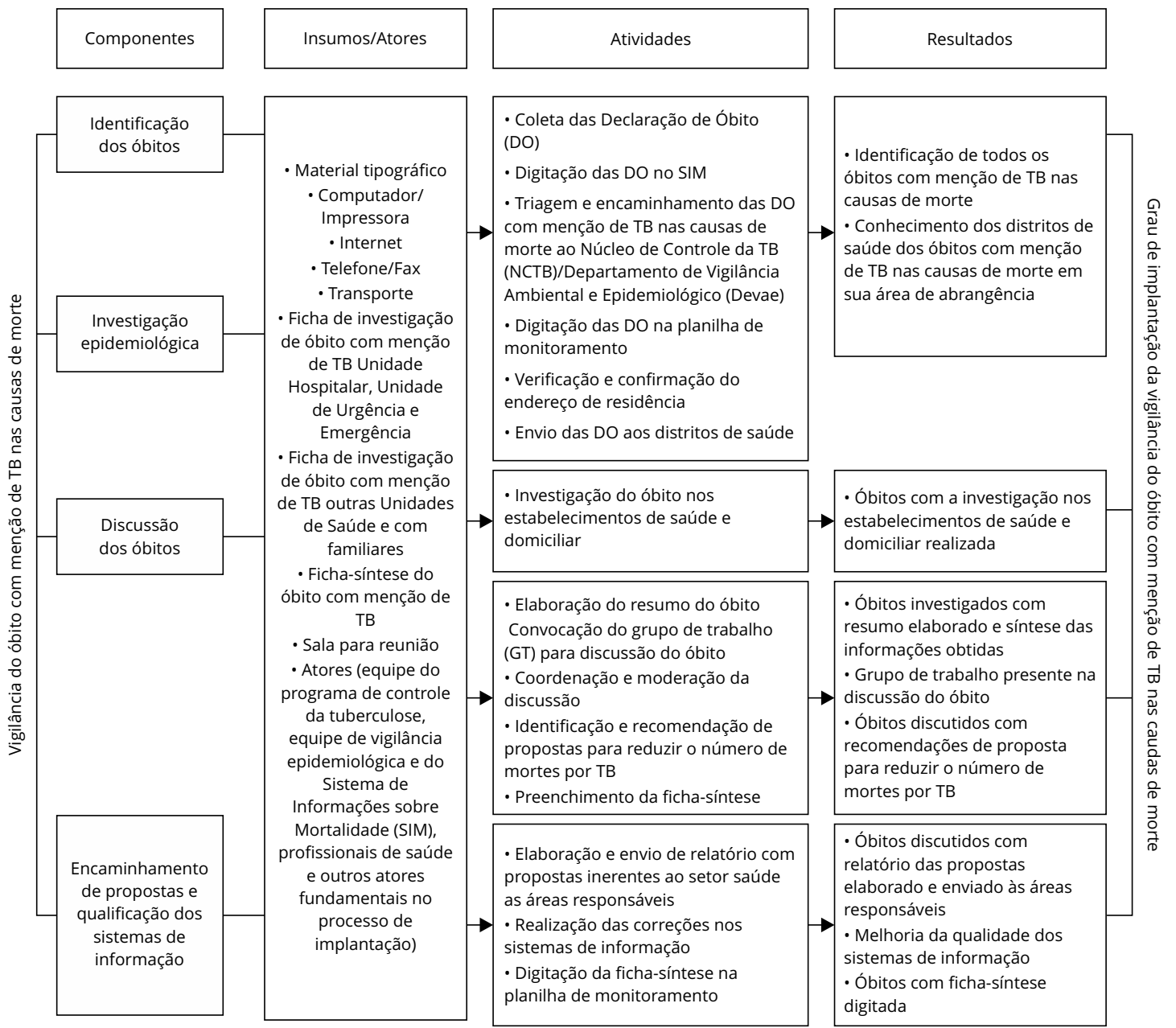

bramento da investigação e discussão dos óbitos (que também depende do apoio de outros setores e áreas técnicas). As maiores pontuações foram para os componentes "investigação epidemiológica" e "discussão dos óbitos", entendendo que as iniciativas de investigação do óbito e organização do grupo de trabalho partem dos PCT e são atividades essenciais da implantação da vigilância do óbito com menção de TB nas causas de morte 3 . Buscou-se, dentro de cada componente na abordagem, estrutura e processo, distribuir os pontos proporcionalmente aos indicadores e, quando não foi possível, atribuiu-se maior valor aos indicadores que o Programa Municipal de Controle da Tuberculose (PMCT) tivesse mais governabilidade.

Os pontos de corte para definir o grau de implantação da vigilância do óbito com menção de TB nas causas de morte foram divididos em três estratos e distribuídos de acordo com a pontuação obtida em relação à pontuação máxima esperada em cada componente abordado: $<40 \%$ - não implantada; entre $\geq 40$ a $<75 \%$ - implantada parcialmente; $\geq 75 \%$ - implantada.

Para análise dos efeitos da adoção do protocolo, além de considerados os indicadores de resultados por componente, foram calculadas as taxas de incidência e mortalidade por TB. 


\section{Quadro 1}

Matriz de indicadores e parâmetros por componentes e abordagens da vigilância do óbito com menção de tuberculose (TB)

\begin{tabular}{|c|c|c|c|c|}
\hline COMPONENTES & ABORDAGENS & INDICADORES & PARÂMETRO & PONTUAÇÃO \\
\hline \multirow[t]{10}{*}{$\begin{array}{l}\text { Indicação dos } \\
\text { óbitos }\end{array}$} & \multirow[t]{5}{*}{ Estrutura } & $\begin{array}{c}\text { Técnico qualificado em nível central para alimentação } \\
\text { das DO com menção de TB no SIM }\end{array}$ & Sim & $\operatorname{Sim}=1 ;$ Não $=0$ \\
\hline & & Dispõe de transporte para as atividades do SIM & Sim & $\operatorname{Sim}=1 ;$ Não = 0 \\
\hline & & Dispõe de computadores para as atividades do SIM & Sim & $\operatorname{Sim}=1 ;$ Não $=0$ \\
\hline & & Dispões de impressora com copiadora & Sim & $\operatorname{Sim}=1 ;$ Não = 0 \\
\hline & & Internet instalada & $\begin{array}{l}\text { Acima de } 15 \\
\text { Mbps }\end{array}$ & $\begin{array}{c}\text { Acima de } 15 \mathrm{Mbps}=1 ; 11-15 \mathrm{Mbps}= \\
0,75 ; 6-10 \mathrm{Mbps}=0,50 ; 1-5 \mathrm{Mbps}=0,25 ; \\
<1 \mathrm{Mbps}=0 .\end{array}$ \\
\hline & \multirow[t]{4}{*}{ Processo } & $\begin{array}{l}\text { Tempo médio entre a ocorrência do óbito e } \\
\text { recebimento da DO }\end{array}$ & $\leq 7$ dias & $\leq 7$ dias $=1 ;>7$ dias $=0$ \\
\hline & & $\begin{array}{l}\text { Tempo médio entre a ocorrência do óbito e } \\
\text { alimentação da DO no SIM }\end{array}$ & $\leq 60$ dias & $\leq 60$ dias $=1 ;>60$ dias $=0$ \\
\hline & & $\begin{array}{l}\text { Realiza triagem das DO com menção de TB nas } \\
\text { causas de morte }\end{array}$ & Sim & $\operatorname{Sim}=2 ;$ Não $=0$ \\
\hline & & $\begin{array}{l}\text { Encaminha as DO com menção de TB nas causas de } \\
\text { morte ao NCTB }\end{array}$ & $80-100 \%$ & $\begin{array}{c}80-100 \%=1 ; 60-79 \%=0,75 ; 40-59 \%= \\
0,50 ; 20-39 \%=0,25 \text { e }<20 \%=0\end{array}$ \\
\hline & Resultado & $\begin{array}{l}\text { Cobertura dos óbitos com menção de TB nas causas } \\
\text { de morte }\end{array}$ & $80-100 \%$ & - \\
\hline \multirow[t]{11}{*}{$\begin{array}{l}\text { Investigação } \\
\text { epidemiológica }\end{array}$} & \multirow[t]{6}{*}{ Estrutura } & $\begin{array}{l}\text { \% profissionais da equipe do PCT que participaram } \\
\text { de capacitação, treinamento, curso ou orientação } \\
\text { sobre investigação do óbito com menção de TB }\end{array}$ & $80-100 \%$ & $\begin{array}{c}80-100 \%=3 ; 60-79 \%=2,25 ; 40-59 \%= \\
1,50 ; 20-39 \%=0,75 \text { e }<20 \%=0\end{array}$ \\
\hline & & $\begin{array}{c}\text { \% profissionais da equipe da vigilância } \\
\text { epidemiológica que participaram de capacitação, } \\
\text { treinamento, curso ou orientação sobre investigação } \\
\text { do óbito com menção de TB }\end{array}$ & $80-100 \%$ & $\begin{array}{c}80-100 \%=3 ; 60-79 \%=2,25 ; 40-59 \%= \\
1,50 ; 20-39 \%=0,75 \text { e }<20 \%=0\end{array}$ \\
\hline & & $\begin{array}{c}\text { \% de NVEH que participaram de capacitação, } \\
\text { treinamento, curso ou orientação sobre investigação } \\
\text { do óbito com menção de TB }\end{array}$ & $80-100 \%$ & $\begin{array}{c}80-100 \%=3 ; 60-79 \%=2,25 ; 40-59 \%= \\
1,50 ; 20-39 \%=0,75 \text { e }<20 \%=0\end{array}$ \\
\hline & & $\begin{array}{l}\text { Possui ficha de investigação de óbito com menção de } \\
\text { TB hospitalar * }\end{array}$ & Sim & $\begin{array}{c}80-100 \%=3 ; 60-79 \%=2,25 ; 40-59 \%= \\
1,50 ; 20-39 \%=0,75 \text { e }<20 \%=0\end{array}$ \\
\hline & & $\begin{array}{c}\text { Possui ficha de investigação de óbito com menção de } \\
\text { TB outras Unidades de Saúde e com familiares* }\end{array}$ & Sim & $\begin{array}{c}80-100 \%=3 ; 60-79 \%=2,25 ; 40-59 \%= \\
1,50 ; 20-39 \%=0,75 \text { e }<20 \%=0\end{array}$ \\
\hline & & $\begin{array}{l}\text { Dispõe de transporte para executar as investigações } \\
\text { de óbito com menção de TB * }\end{array}$ & Sim & $\begin{array}{c}80-100 \%=3 ; 60-79 \%=2,25 ; 40-59 \%= \\
1,50 ; 20-39 \%=0,75 \text { e }<20 \%=0\end{array}$ \\
\hline & \multirow[t]{5}{*}{ Processo } & $\begin{array}{l}\text { Utiliza ficha de investigação de óbito com menção de } \\
\qquad \text { TB hospitalar * }\end{array}$ & Sim & $\begin{array}{c}80-100 \%=3 ; 60-79 \%=2,25 ; 40-59 \%= \\
1,50 ; 20-39 \%=0,75 \text { e }<20 \%=0\end{array}$ \\
\hline & & $\begin{array}{c}\text { Utiliza ficha de investigação de óbito com menção de } \\
\text { TB outras Unidades de Saúde e com familiares* }\end{array}$ & Sim & $\begin{array}{c}80-100 \%=3 ; 60-79 \%=2,25 ; 40-59 \%= \\
1,50 ; 20-39 \%=0,75 \text { e }<20 \%=0\end{array}$ \\
\hline & & $\begin{array}{l}\text { \% profissionais da equipe do PCT que realizam } \\
\text { investigação de óbito com menção de TB }\end{array}$ & $80-100 \%$ & $\begin{array}{c}80-100 \%=3 ; 60-79 \%=2,25 ; 40-59 \%= \\
1,50 ; 20-39 \%=0,75 \text { e }<20 \%=0\end{array}$ \\
\hline & & $\begin{array}{l}\text { \% profissionais da equipe da vigilância } \\
\text { epidemiológica que realizam investigação de óbito } \\
\text { com menção de TB }\end{array}$ & $80-100 \%$ & $\begin{array}{c}80-100 \%=3 ; 60-79 \%=2,25 ; 40-59 \%= \\
1,50 ; 20-39 \%=0,75 \text { e }<20 \%=0\end{array}$ \\
\hline & & $\begin{array}{c}\text { \% dos NVEH que estão enviando a investigação } \\
\text { hospitalar dos óbitos ocorridos no seu } \\
\text { estabelecimento }\end{array}$ & $80-100 \%$ & $\begin{array}{c}80-100 \%=2 ; 60-79 \%=1,50 ; 40-59 \%= \\
1 ; 20-39 \%=0,50 \text { e }<20 \%=0\end{array}$ \\
\hline
\end{tabular}


Quadro 1 (continuação)

\begin{tabular}{|c|c|c|c|c|}
\hline COMPONENTES & ABORDAGENS & INDICADORES & PARÂMETRO & PONTUAÇÃO \\
\hline \multirow[t]{4}{*}{$\begin{array}{l}\text { Investigação } \\
\text { epidemiológica }\end{array}$} & \multirow[t]{3}{*}{ Processo } & $\begin{array}{l}\text { \% de óbitos com fichas de investigação hospitalar } \\
\text { enviadas pelos NVEH }\end{array}$ & $80-100 \%$ & $\begin{array}{c}80-100 \%=2 ; 60-79 \%=1,50 ; 40-59 \%= \\
1 ; 20-39 \%=0,50 \text { e }<20 \%=0\end{array}$ \\
\hline & & $\begin{array}{l}\text { \% de óbitos com ficha de investigação na Unidade de } \\
\text { Saúde que o paciente foi atendido }\end{array}$ & $80-100 \%$ & \multirow[t]{2}{*}{$\begin{array}{c}80-100 \%=3 ; 60-79 \%=2,25 ; 40-59 \%= \\
\quad 1,50 ; 20-39 \%=0,75 \text { e }<20 \%=0\end{array}$} \\
\hline & & \% de óbitos com as fichas de investigação domiciliar & $80-100 \%$ & \\
\hline & Resultados & $\begin{array}{c}\% \text { de óbitos investigados entre o total de óbitos com } \\
\text { a TB nas causas de morte no período analisado }\end{array}$ & $\begin{array}{l}\text { Pelo menos } \\
\quad 25 \%\end{array}$ & - \\
\hline \multirow[t]{20}{*}{$\begin{array}{l}\text { Discussão dos } \\
\text { óbitos }\end{array}$} & \multirow[t]{7}{*}{ Estrutura } & Dispõe de ficha-síntese de conclusão do caso * & Sim & $\begin{array}{c}80-100 \%=2 ; 60-79 \%=1,50 ; 40-59 \%= \\
1 ; 20-39 \%=0,50 \text { e }<20 \%=0\end{array}$ \\
\hline & & $\begin{array}{l}\text { Dispõe de notebook ou computador para realizar as } \\
\text { atividades de discussão dos óbitos * }\end{array}$ & Sim & $\begin{array}{c}80-100 \%=2 ; 60-79 \%=1,50 ; 40-59 \%= \\
1 ; 20-39 \%=0,50 \text { e }<20 \%=0\end{array}$ \\
\hline & & $\begin{array}{l}\text { Dispõe de projetor para realizar as atividades de } \\
\text { discussão dos óbitos * }\end{array}$ & Sim & $\begin{array}{c}80-100 \%=2 ; 60-79 \%=1,50 ; 40-59 \%= \\
1 ; 20-39 \%=0,50 \text { e }<20 \%=0\end{array}$ \\
\hline & & Internet instalada * & $\begin{array}{l}\text { Acima de } \\
15 \mathrm{Mbps}\end{array}$ & $\begin{array}{c}80-100 \%=2 ; 60-79 \%=1,50 ; 40-59 \%= \\
1 ; 20-39 \%=0,50 \text { e }<20 \%=0\end{array}$ \\
\hline & & $\begin{array}{l}\text { Possui impressora com copiadora para realizar as } \\
\text { atividades de discussão dos óbitos nos DS * }\end{array}$ & Sim & $\begin{array}{c}80-100 \%=1 ; 60-79 \%=0,75 ; 40-59 \%= \\
0,50 ; 20-39 \%=0,25 \text { e }<20 \%=0\end{array}$ \\
\hline & & Dispõe de telefone ou fax * & Sim & $\begin{array}{c}80-100 \%=1 ; 60-79 \%=0,75 ; 40-59 \%= \\
0,50 ; 20-39 \%=0,25 \text { e }<20 \%=0\end{array}$ \\
\hline & & $\begin{array}{l}\text { Existe sala de reunião para o grupo de trabalho } \\
\text { discutir o óbito * }\end{array}$ & Sim & $\begin{array}{c}80-100 \%=2 ; 60-79 \%=1,50 ; 40-59 \%= \\
1 ; 20-39 \%=0,50 \text { e }<20 \%=0\end{array}$ \\
\hline & \multirow[t]{10}{*}{ Processo } & Convoca grupo de trabalho para discussão dos casos & Sim & $\operatorname{Sim}=2 ;$ Não $=0$ \\
\hline & & Documenta as reuniões de discussão dos óbitos & ATA & $\operatorname{Sim}=2 ;$ Não $=0$ \\
\hline & & $\begin{array}{l}\text { \% de discussão dos óbitos com participação da } \\
\text { equipe do PCT estadual }\end{array}$ & $80-100 \%$ & $\begin{array}{c}80-100 \%=1 ; 60-79 \%=0,75 ; 40-59 \%= \\
0,50 ; 20-39 \%=0,25 \text { e }<20 \%=0\end{array}$ \\
\hline & & $\begin{array}{l}\text { \% de discussão dos óbitos com participação da } \\
\text { equipe do PCT municipal }\end{array}$ & $80-100 \%$ & $\begin{array}{c}80-100 \%=2 ; 60-79 \%=1,50 ; 40-59 \%= \\
1 ; 20-39 \%=0,50 \text { e }<20 \%=0\end{array}$ \\
\hline & & $\begin{array}{c}\text { \% de discussão dos óbitos com participação da } \\
\text { equipe da vigilância epidemiológica }\end{array}$ & $80-100 \%$ & $\begin{array}{c}80-100 \%=2 ; 60-79 \%=1,50 ; 40-59 \%= \\
1 ; 20-39 \%=0,50 \text { e }<20 \%=0\end{array}$ \\
\hline & & $\begin{array}{l}\text { \% de discussão dos óbitos com participação dos } \\
\text { profissionais dos estabelecimentos de saúde }\end{array}$ & $80-100 \%$ & $\begin{array}{c}80-100 \%=2 ; 60-79 \%=1,50 ; 40-59 \%= \\
1 ; 20-39 \%=0,50 \text { e }<20 \%=0\end{array}$ \\
\hline & & $\begin{array}{l}\text { \% discussão dos óbitos com participação dos } \\
\text { profissionais dos NVEH }\end{array}$ & $80-100 \%$ & $\begin{array}{c}80-100 \%=1 ; 60-79 \%=0,75 ; 40-59 \%= \\
0,50 ; 20-39 \%=0,25 \text { e }<20 \%=0\end{array}$ \\
\hline & & $\begin{array}{l}\% \text { discussão dos óbitos com participação dos } \\
\text { gestores }\end{array}$ & $80-100 \%$ & $\begin{array}{c}80-100 \%=2 ; 60-79 \%=1,50 ; 40-59 \%= \\
1 ; 20-39 \%=0,50 \text { e }<20 \%=0\end{array}$ \\
\hline & & $\begin{array}{l}\text { Elabora ficha-síntese de conclusão do caso com o } \\
\text { grupo de trabalho de discussão dos óbitos }\end{array}$ & Sim & $\operatorname{Sim}=2 ; \mathrm{Não}=0$ \\
\hline & & \% óbitos investigados com ficha-síntese preenchida & $80-100 \%$ & $\begin{array}{c}80-100 \%=2 ; 60-79 \%=1,50 ; 40-59 \%= \\
1 ; 20-39 \%=0,50 \text { e }<20 \%=0\end{array}$ \\
\hline & \multirow[t]{3}{*}{ Resultados } & $\begin{array}{l}\% \text { de óbitos discutidos com grupo de trabalho } \\
\text { formado }\end{array}$ & $\geq 25 \%$ & - \\
\hline & & $\begin{array}{c}\text { \% de óbitos discutidos com os gestores, equipe } \\
\text { técnica da atenção básica, da vigilância em saúde e } \\
\text { da vigilância hospitalar e com a sociedade civil, entre } \\
\text { outras representações estratégicas. }\end{array}$ & $\geq 25 \%$ & - \\
\hline & & $\begin{array}{l}\text { Tempo médio entre a data do óbito e o } \\
\text { encerramento da discussão do caso }\end{array}$ & $<120$ dias & - \\
\hline
\end{tabular}

(continua) 


\begin{tabular}{|c|c|c|c|c|}
\hline COMPONENTES & ABORDAGENS & INDICADORES & PARÂMETRO & PONTUAÇÃO \\
\hline \multirow{11}{*}{$\begin{array}{l}\text { Encaminha- } \\
\text { mento de } \\
\text { propostas e } \\
\text { qualificação } \\
\text { dos sistemas de } \\
\text { informação }\end{array}$} & \multirow[t]{8}{*}{ Estrutura } & $\begin{array}{c}\text { Técnico qualificado para realizar as alterações no } \\
\text { SINAN sugeridas pelo grupo de trabalho a partir da } \\
\text { ficha-síntese }\end{array}$ & Sim & $\operatorname{Sim}=4 ; N a ̃ o=0$ \\
\hline & & $\begin{array}{c}\text { Técnico qualificado para realizar as alterações no SIM } \\
\text { sugeridas pelo grupo de trabalho a partir da ficha- } \\
\text { síntese }\end{array}$ & Sim & $\operatorname{Sim}=4 ;$ Não $=0$ \\
\hline & & $\begin{array}{l}\text { \% de óbitos com alteração do tipo de entrada no } \\
\text { SINAN após a discussão do caso }\end{array}$ & $80-100 \%$ & $\begin{array}{c}80-100 \%=4 ; 60-79 \%=3 ; 40-59 \%=2 ; \\
20-39 \%=1 \text { e }<20 \%=0\end{array}$ \\
\hline & & $\begin{array}{c}\% \text { de óbitos com informação dos exames } \\
\text { laboratoriais qualificada no SINAN após a discussão } \\
\text { do caso }\end{array}$ & $80-100 \%$ & $\begin{array}{c}80-100 \%=4 ; 60-79 \%=3 ; 40-59 \%=2 ; \\
20-39 \%=1 \text { e }<20 \%=0\end{array}$ \\
\hline & & $\begin{array}{l}\text { \% de óbitos com situação de encerramento alterada } \\
\text { após a discussão do caso }\end{array}$ & $80-100 \%$ & $\begin{array}{c}80-100 \%=4 ; 60-79 \%=3 ; 40-59 \%=2 ; \\
20-39 \%=1 \text { e }<20 \%=0\end{array}$ \\
\hline & & $\begin{array}{l}\text { \% de óbitos com alteração nas causas do óbito no } \\
\text { SIM após a discussão do caso }\end{array}$ & $80-100 \%$ & $\begin{array}{c}80-100 \%=4 ; 60-79 \%=3 ; 40-59 \%=2 ; \\
20-39 \%=1 \text { e }<20 \%=0\end{array}$ \\
\hline & & $\begin{array}{l}\text { \% de óbitos com alteração do código A16 (CID-10) } \\
\text { referente à tuberculose sem realização de exame } \\
\text { para diagnóstico para um código (A15, A17 a A19) no } \\
\text { qual consta a confirmação diagnóstica }\end{array}$ & $80-100 \%$ & $\begin{array}{c}80-100 \%=4 ; 60-79 \%=3 ; 40-59 \%=2 ; \\
20-39 \%=1 \text { e }<20 \%=0\end{array}$ \\
\hline & & $\begin{array}{l}\text { \% de óbitos com outras alterações realizadas no SIM } \\
\text { após a discussão do caso }\end{array}$ & $80-100 \%$ & $\begin{array}{c}80-100 \%=4 ; 60-79 \%=3 ; 40-59 \%=2 ; \\
20-39 \%=1 \text { e }<20 \%=0\end{array}$ \\
\hline & \multirow[t]{3}{*}{ Resultado } & $\begin{array}{c}\% \text { de óbitos discutidos e com relatórios enviados } \\
\text { para os estabelecimentos envolvidos }\end{array}$ & $80-100 \%$ & - \\
\hline & & $\begin{array}{l}\text { \% de óbitos com menção de TB registrados no SIM e } \\
\text { não notificado no SINAN entre o total de óbitos com } \\
\text { menção de tuberculose registrados no SIM }\end{array}$ & $80-100 \%$ & - \\
\hline & & $\begin{array}{c}\text { \% de óbitos com confirmação diagnóstica e } \\
\text { registrada no SIM com código A16 na causa básica ou } \\
\text { associada }\end{array}$ & $0 \%$ & - \\
\hline
\end{tabular}

CID-10: Classificação Internacional de Doenças, 10ạ revisão; DO: Declaração de Óbito; DS: distritos sanitários; Mbps: megabites por segundo; NCTB: Núcleo de Controle da TB; NVEH: Núcleo Vigilância Epidemiológico Hospitalar PCT: Programas de Controle da TB; SIM: Sistema de Informações sobre Mortalidade; SINAN: Sistema de Informação de Agravos de Notificação.

* Indicadores cujas informações partem de diversos profissionais (coordenação municipal do PCT e os responsáveis técnicos pelo PCT a nível distrital), os valores foram somados e foi calculada a média.

$\mathrm{Na}$ análise das taxas de incidência por TB, foi utilizado software Stata/SE 13.0 (https://www.stata. com) para correção das duplicidades de casos de TB registradas no SINAN. As variáveis "nome do paciente", "nome da mãe" e "data de nascimento" foram adotadas como variáveis de pareamento. Ao identificar duplicidade verdadeira e duplo registro durante o mesmo tratamento, apenas uma ficha de notificação foi mantida 6 . Foram incluídos na análise os casos novos de TB todas as formas, ano diagnóstico 2005 a 2019, e excluídos os encerrados por mudança de diagnóstico. Os dados com as frequências foram organizados no programa Microsoft Excel 2003 (https://products.office.com/) e foram calculadas as taxas brutas e padronizadas da incidência e mortalidade por TB. A proporção dos óbitos por TB pulmonar com e sem confirmação laboratorial utilizando fórmula padrão para óbitos com confirmação laboratorial: (número de óbitos de residentes por TB causa básica no período com confirmação diagnóstica/total de óbitos de residentes por TB causa básica no mesmo período X 100), e para os óbitos sem confirmação laboratorial, foi utilizada a mesma fórmula, entretanto, 
no numerador, o número de óbitos de residentes por TB causa básica no período sem confirmação diagnóstica para TB. A categoria A16 da CID-10 foi usada para classificar a TB das vias respiratórias sem confirmação bacteriológica ou histológica e a categoria A15, para classificar TB respiratória com confirmação por exame microscópio da expectoração, com ou sem cultura.

As taxas foram padronizadas pelo método direto, utilizando no numerador os casos novos e óbitos por TB, e o denominador foi a população residente de Manaus do Censo Demográfico de 2010 como padrão. A evolução temporal foi apresentada em figuras acrescida de informação do modelo de regressão simples para incidência e de variação percentual para a mortalidade.

Após identificação da normalidade das taxas de incidência, por meio do teste Shapiro-Wilk, foi realizada análise de regressão linear simples, tendo como variável dependente a taxa e os anos como variáveis independentes. Para a significância estatística, considerou-se valor de $\mathrm{p}<5 \%$, e o ajuste do modelo foi avaliado por meio do R2 . A taxa de mortalidade não apresentou distribuição normal. Nesse caso, avaliou-se somente percentual de variação, a fim de julgar se houve crescimento da taxa de mortalidade, considerando os seguintes agrupamentos em anos: 2006-2009, 2010-2013, 2014- 2016 e 2017-2019. Esses agrupamentos foram definidos a partir da inspeção dos dados e do conhecimento histórico da epidemiologia da doença na cidade, assim como a evidenciação do período de implantação da vigilância do óbito com menção de TB. Nesta etapa, utilizou-se o software Stata/SE 13.0 para análise.

O projeto foi aprovado no Comitê de Ética em Pesquisa da Universidade Federal do Amazonas (CEP/UFAM, parecer no 3.729.611), sendo respeitados os requisitos éticos da Resolução no 466/2012 do Conselho Nacional de Saúde.

\section{Resultados}

O questionário foi respondido por sete profissionais fontes de informação: o coordenador municipal do PCT, os responsáveis técnicos do PCT em nível distrital (um de cada DS - Norte, Sul, Leste, Oeste e Rural) e pelo técnico responsável pela gestão do SIM. A coleta dos dados ocorreu nos meses de dezembro 2019 a janeiro de 2020. Após contato prévio e agendamento com os respondentes, foi explicada a pesquisa, obtido Termo de Consentimento Livre e Esclarecido (TCLE) e entregue o questionário para posterior devolução, o qual foi respondido com média de quatro dias. A completude dos campos do questionário respondido pelos profissionais do PCT municipal foi 100\% e, do técnico responsável pela gestão do SIM, $80 \%$.

O resultado da avaliação de implantação da vigilância de óbito com menção de TB mostrou que essa implantação se diferenciou conforme os componentes necessários à operacionalização do referido protocolo.

O grau de implantação do componente "identificação dos óbitos" alcançou 65\% do resultado esperado, sendo classificado como parcialmente implantado. Nesse componente, a abordagem "estrutura" ficou $50 \%$ aquém do resultado esperado. O resultado desse componente foi principalmente afetado pela falta de técnico qualificado em nível central para vigilância do óbito de TB para alimentação das Declarações de Óbito (DO) com menção de TB no SIM, e pela falta de transporte institucional para atividades do SIM. A abordagem dos indicadores de "processo" alcançou 80\% do resultado esperado, com três indicadores obtendo o valor máximo, são eles: o tempo médio entre a ocorrência do óbito e a alimentação da DO no SIM, a triagem das DO com menção de TB nas causas de morte e o encaminhamento das DO com menção de TB ao PCT municipal (Tabela 1). 
Tabela 1

Resultado da matriz de julgamento da vigilância do óbito com menção de tuberculose (TB). Manaus, Amazonas, Brasil, 2017 a 2019.

\begin{tabular}{|c|c|c|c|c|c|}
\hline \multirow[t]{2}{*}{ Componentes } & \multirow[t]{2}{*}{ Indicadores } & \multirow[t]{2}{*}{ Valores contratados } & \multirow[t]{2}{*}{ Pontos máximo } & \multicolumn{2}{|c|}{$\Sigma$ da pontuação obtida } \\
\hline & & & & $\mathbf{N}$ & $\%$ \\
\hline Identificação & Abordagem estrutura & & & & \\
\hline \multirow[t]{17}{*}{ dos óbitos } & Técnico qualificado em nível central para & Não & 1 & 0 & 0,0 \\
\hline & alimentação das DO com menção de TB no SIM & & & & \\
\hline & Dispõe de transporte para as atividades do SIM & Não & 1 & 0 & 0,0 \\
\hline & Dispõe de computadores para as atividades do & Sim & 1 & 1 & 100,0 \\
\hline & SIM & & & & \\
\hline & Dispões de impressora com copiadora & Sim & 1 & 1 & 100,0 \\
\hline & Internet instalada & 6-10Mbps & 1 & 0,50 & 50,0 \\
\hline & Subtotal & & 5 & 2,5 & 50,0 \\
\hline & Abordagem processo & & & & \\
\hline & $\begin{array}{l}\text { Tempo médio entre a ocorrência do óbito e } \\
\text { recebimento da DO }\end{array}$ & $>7$ dias & 1 & 0 & 0,0 \\
\hline & $\begin{array}{l}\text { Tempo médio entre a ocorrência do óbito e } \\
\text { alimentação da DO no SIM }\end{array}$ & $\leq 60$ dias & 1 & 1 & 100,0 \\
\hline & $\begin{array}{l}\text { Realiza triagem das DO com menção de TB nas } \\
\text { causas de morte }\end{array}$ & Sim & 2 & 2 & 100,0 \\
\hline & $\begin{array}{l}\text { Encaminha as DO com menção de TB nas causas } \\
\text { de morte ao NCTB }\end{array}$ & Sim & 1 & 1 & 100,0 \\
\hline & Subtotal & & 5 & 4 & 80,0 \\
\hline & Grau de implantação do componente & & 10 & 6,5 & 65,0 \\
\hline & (estrutura e processo) & & & & \\
\hline & & & & & $\begin{array}{l}\text { (implantado } \\
\text { parcialmente) }\end{array}$ \\
\hline Investigação & Abordagem estrutura & & & & \\
\hline \multirow[t]{13}{*}{ epidemiológica } & $\%$ profissionais da equipe do PMCT que & $40,0-59,0$ & 3 & 1,5 & 50,0 \\
\hline & participaram de capacitação, treinamento, curso & & & & \\
\hline & ou orientação sobre investigação do óbito com & & & & \\
\hline & menção de TB & & & & \\
\hline & $\%$ profissionais dos NVEH que participaram de & $40,0-59,0$ & 3 & 1,5 & 50,0 \\
\hline & capacitação, treinamento, curso ou orientação & & & & \\
\hline & sobre investigação do óbito com menção de TB & & & & \\
\hline & $\begin{array}{l}\text { \% de NVEH que participaram de capacitação, } \\
\text { treinamento, curso ou orientação sobre }\end{array}$ & $60,0-79,0$ & 3 & 2,25 & 75,0 \\
\hline & investigação do óbito com menção de TB & & & & \\
\hline & $\begin{array}{l}\text { Possui ficha de investigação de óbito com } \\
\text { menção de TB hospitalar }\end{array}$ & $80,0-100,0$ & 3 & 3 & 100,0 \\
\hline & Possui ficha de investigação de óbito com & $80,0-100,0$ & 3 & 3 & 100,0 \\
\hline & $\begin{array}{l}\text { menção de TB outras Unidades de Saúde e com } \\
\text { familiares }\end{array}$ & & & & \\
\hline & $\begin{array}{l}\text { Dispõe de transporte para executar as } \\
\text { investigações de óbito com menção de TB }\end{array}$ & $80,0-100,0$ & 3 & 3 & 100,0 \\
\hline
\end{tabular}

(continua) 
Tabela 1 (continuação)

\begin{tabular}{|c|c|c|c|c|c|}
\hline \multirow[t]{2}{*}{ Componentes } & \multirow[t]{2}{*}{ Indicadores } & \multirow[t]{2}{*}{ Valores contratados } & \multirow[t]{2}{*}{ Pontos máximo } & \multicolumn{2}{|c|}{$\Sigma$ da pontuação obtida } \\
\hline & & & & $\mathbf{N}$ & $\%$ \\
\hline Investigação & Subtotal & & 18 & 14 & 79,2 \\
\hline \multirow[t]{11}{*}{ epidemiológica } & Abordagem processo & & & & \\
\hline & $\begin{array}{l}\text { \% que utiliza ficha de investigação de óbito com } \\
\text { menção de TB hospitalar }\end{array}$ & $80,0-100,0$ & 3 & 3 & 100,0 \\
\hline & $\begin{array}{l}\% \text { que utiliza ficha de investigação de óbito com } \\
\text { menção de TB outras Unidades de Saúde e com } \\
\text { familiares }\end{array}$ & $80,0-100,0$ & 3 & 3 & 100,0 \\
\hline & $\begin{array}{l}\text { \% profissionais da equipe do PMCT que realizam } \\
\text { investigação de óbito com menção de TB }\end{array}$ & $20,0-39,0$ & 3 & 0,75 & 25,0 \\
\hline & $\begin{array}{l}\text { \% profissionais da equipe da vigilância } \\
\text { epidemiológica que realizam investigação de } \\
\text { óbito com menção de TB }\end{array}$ & $<20,0$ & 3 & 0,0 & 0,0 \\
\hline & $\begin{array}{l}\text { \% dos NVEH que estão enviando a investigação } \\
\text { hospitalar dos óbitos ocorridos no seu } \\
\text { estabelecimento }\end{array}$ & $20,0-39,0$ & 2 & 0,5 & 25,0 \\
\hline & $\begin{array}{l}\% \text { de óbitos com ficha de investigação hospitalar } \\
\text { enviadas pelos } \mathrm{NVEH}\end{array}$ & $20,0-39,0$ & 2 & 0,5 & 25,0 \\
\hline & $\begin{array}{l}\text { \% de óbitos com ficha de investigação na } \\
\text { unidade de saúde que o paciente foi atendido }\end{array}$ & $<20,0$ & 3 & 0 & 0,0 \\
\hline & $\begin{array}{l}\text { \% de óbitos com as fichas de investigação } \\
\text { domiciliar }\end{array}$ & $<20,0$ & 3 & 0 & 0,0 \\
\hline & Subtotal & & 22 & 7,75 & 35,2 \\
\hline & $\begin{array}{l}\text { Grau de implantação do componente } \\
\text { (estrutura e processo) }\end{array}$ & & 40 & 22 & $\begin{array}{l}55,0 \\
\text { (implantado } \\
\text { parcialmente) }\end{array}$ \\
\hline Discussão dos & Abordagem estrutura & & & & \\
\hline \multirow[t]{7}{*}{ óbitos } & Dispõe de ficha síntese de conclusão do caso & $>20,0$ & 2 & 0 & 0,0 \\
\hline & $\begin{array}{l}\text { Dispõe de notebook ou computador para } \\
\text { realizar as atividades de discussão dos óbitos }\end{array}$ & $80,0-100,0$ & 2 & 2 & 100,0 \\
\hline & $\begin{array}{l}\text { Dispõe de projetor para realizar as atividades de } \\
\text { discussão dos óbitos }\end{array}$ & $80,0-100,0$ & 2 & 2 & 100,0 \\
\hline & Internet instalada & $80,0-100,0$ & 2 & 2 & 100,0 \\
\hline & $\begin{array}{l}\text { Possui impressora com copiadora para realizar } \\
\text { as atividades de discussão dos óbitos nos DS }\end{array}$ & $20,0-39,0$ & 1 & 0,25 & 25,0 \\
\hline & Dispõe de telefone ou fax & $80,0-100,0$ & 1 & 1 & 100,0 \\
\hline & $\begin{array}{l}\text { Existe sala de reunião para o grupo de trabalho } \\
\text { discutir o óbito }\end{array}$ & $60,0-79,0$ & 2 & 1,50 & 75,0 \\
\hline
\end{tabular}

(continua) 
Tabela 1 (continuação)

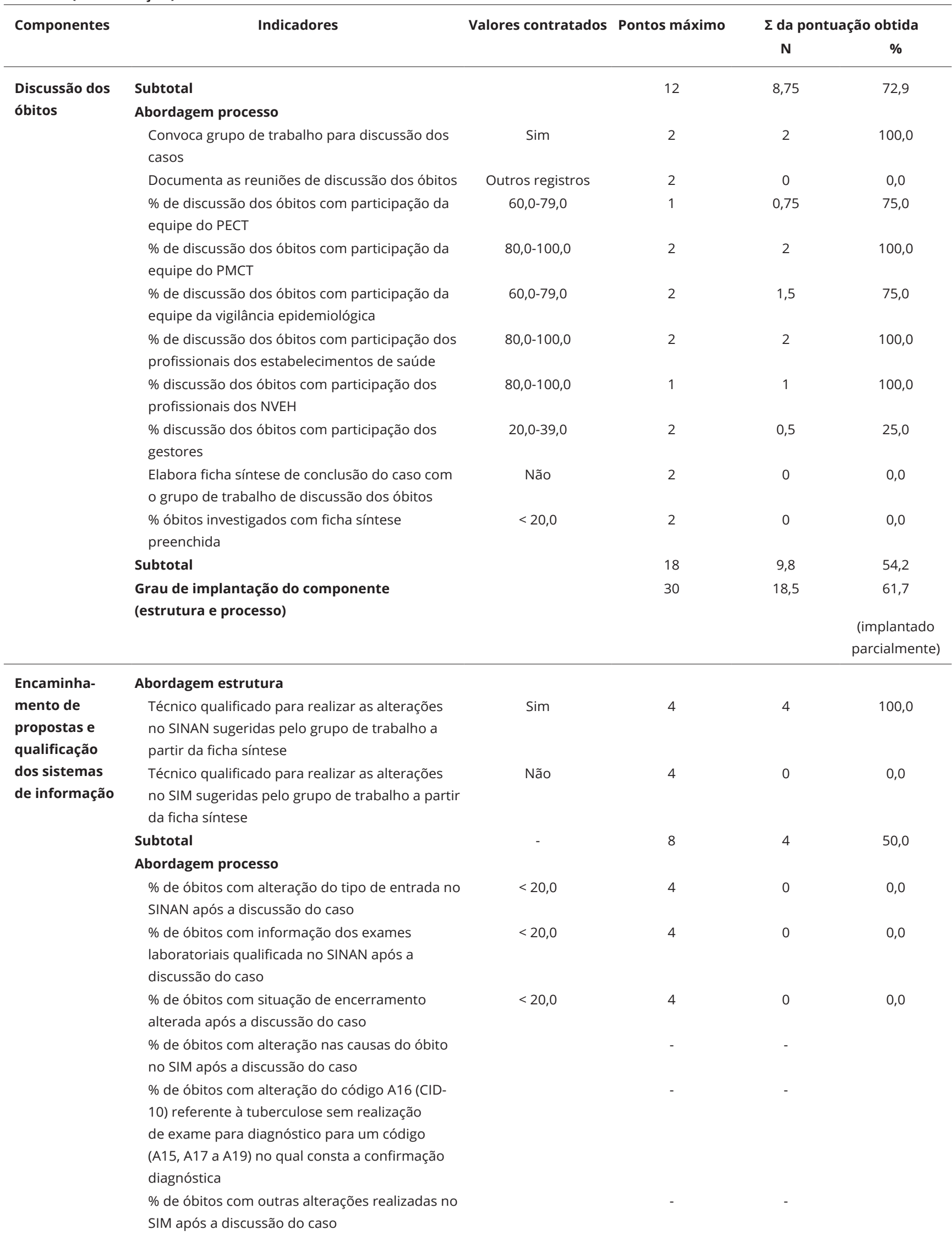

(continua) 
Tabela 1 (continuação)

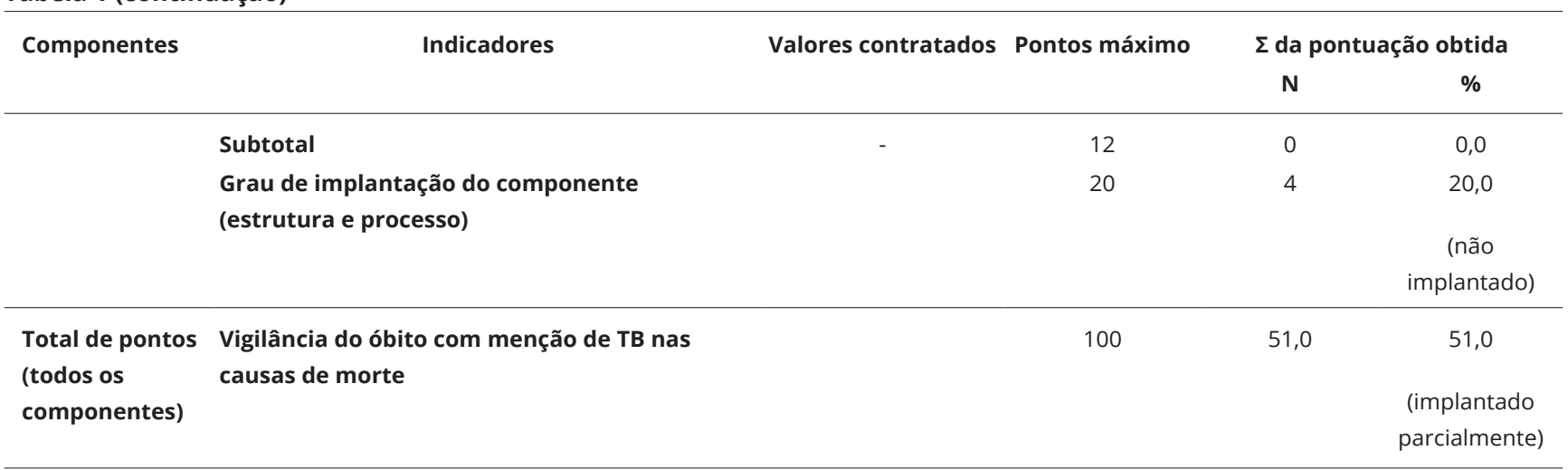

CID-10: Classificação Internacional de Doenças, 10ạ revisão; DO: Declaração de Óbito; DS: distritos sanitários; Mbps: megabites por segundo; NCTB: Núcleo de Controle da Tuberculose; NVEH: Núcleo Vigilância Epidemiológico Hospitalar PCT: Programas de Controle da TB;

PECT: Programa Estadual de Controle da Tuberculose; РMCT: Programa Municipal de Controle da Tuberculose; SIM: Sistema de Informações sobre Mortalidade; SINAN: Sistema de Informação de Agravos de Notificação; TB: tuberculose.

O componente "investigação epidemiológica" alcançou 55\% do resultado esperado, sendo classificado como parcialmente implantado. Na matriz de julgamento do componente "investigação epidemiológica", a abordagem de "estrutura" alcançou 79,2\%, indicando boa estrutura para realizar a investigação epidemiológica. Esse resultado foi medido pelos indicadores que avaliaram a presença de profissionais dos núcleos de vigilância epidemiológica hospitalar (NVEH) que participaram de capacitação, treinamento, curso ou orientação sobre investigação do óbito com menção de TB, pela disponibilidade de ficha de investigação de óbito com menção de TB hospitalar, unidades de saúde e familiar, e pela presença de transporte institucional para executar as investigações com menção de TB nas causas de morte. Os indicadores de "processo" alcançaram 35,2\% do esperado, ocasionado pelo reduzido número de profissionais do PCT de Manaus que realizam investigação de óbito com menção de TB e por não haver profissionais da vigilância epidemiológica que desempenham essa atividade, além do reduzido número de óbitos com ficha de investigação hospitalar preenchida pelos NVEH e número reduzido de óbitos com ficha de investigação preenchida na unidade de saúde e domiciliar (Tabela 1).

O componente "discussão dos óbitos" alcançou 61,7\% do resultado esperado, sendo classificado como parcialmente implantado. Nesse componente, a abordagem "estrutura" alcançou 72,9\% do resultado esperado, por disporem de notebook/computador, telefone/fax, projetor, Internet e sala de reunião para o grupo de trabalho desenvolver as atividades que envolvem a discussão dos óbitos com menção de TB. A abordagem do "processo" desse componente ficou 54,2\%, faltando documentar as reuniões de discussão de óbito e elaborar ficha síntese de conclusão de caso com o grupo de trabalho de discussão dos óbitos (Tabela 1).

O grau de implantação do componente "encaminhamento de propostas e qualificação dos sistemas de informação" foi classificado como não implantado, por alcançar 20\% do resultado esperado. Nesse componente, a abordagem "estrutura" ficou em 50\%, devido à ausência de técnico (codificador) qualificado para vigilância do óbito de TB para realizar as alterações no SIM sugeridas pelo grupo de trabalho. Na abordagem dos indicadores de "processo", as alterações realizadas no SIM após a discussão do caso não foram mensuradas por falta de registro ou impossibilidade de resgate automatizado dessa informação do SIM (Tabela 1).

O grau de implantação da vigilância do óbito com menção de TB nas causas de morte foi considerado parcialmente implantado no PCT de Manaus. Na abordagem dos indicadores de "estrutura", os componentes "identificação do óbito" e "encaminhamento de propostas e qualificação dos sistemas de informação" obtiveram menor percentual. Entre os indicadores de processo, os componentes "investigação epidemiológica" e "encaminhamento de propostas e qualificação dos sistemas de informação" obtiveram menor percentual (Figura 2). 


\section{Figura 2}

Síntese do grau de implantação da vigilância do óbito com menção de tuberculose (TB) a partir dos subtotais do modelo lógico, com acréscimo dos indicadores de resultado. Manaus, Amazonas, Brasil, 2017 a 2019 *.

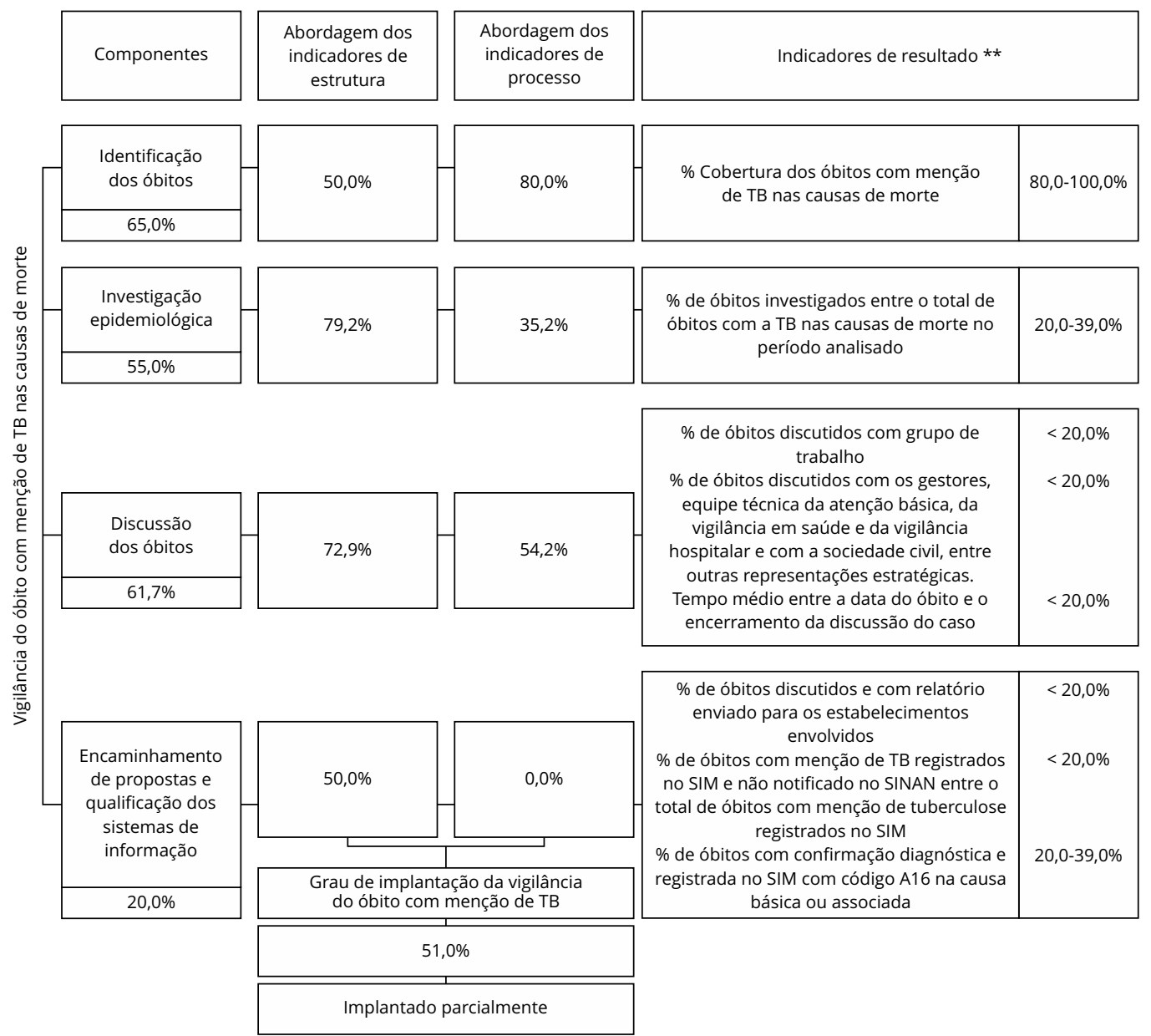

SIM: Sistema de Informações de Mortalidade; SINAN: Sistema de Informação de Agravos de Notificação.

* 2019 - referente ao primeiro semestre.

** Os resultados apresentados no diagrama visam facilitar a compreensão, mas não fizeram parte da matriz de julgamento.

Na Figura 2, além do resumo da matriz de julgamento a partir do modelo lógico, incluíram-se os valores dos indicadores de resultado. Observou-se que, de janeiro de 2017 a julho de 2019, 20\% a $39 \%$ dos óbitos com menção de TB foram investigados e, entre estes, menos de $20 \%$ foram discutidos. Houve inconsistência entre o SIM e SINAN: o óbito por TB registrado no SIM e não notificado no SINAN, e o óbito ter confirmação diagnóstica para TB no SINAN e registrado no SIM na categoria A16 (dados não mostrados).

Quanto à incidência por TB, a partir de 2012, a taxa padronizada da incidência de TB todas as formas afasta-se com relação à taxa bruta, acentuando-se partir de 2017 (Figura 3). No período de 2005 a 2019, houve aumento discreto, porém significativo da taxa padronizada da incidência de TB todas as formas e pulmonar com confirmação laboratorial de $0,23\left(\mathrm{R}^{2}=0,93\right)$ e $0,28\left(\mathrm{R}^{2}=0,91\right)$ casos por $100 \mathrm{mil} /$ habitantes ao ano, respectivamente (valor de $\mathrm{p}<0,01$ ). 
Figura 3

Taxa bruta e padronizada da incidência de tuberculose (TB) todas as formas e pulmonares com confirmação laboratorial. Manaus, Amazonas, Brasil, 2005 a 2019.

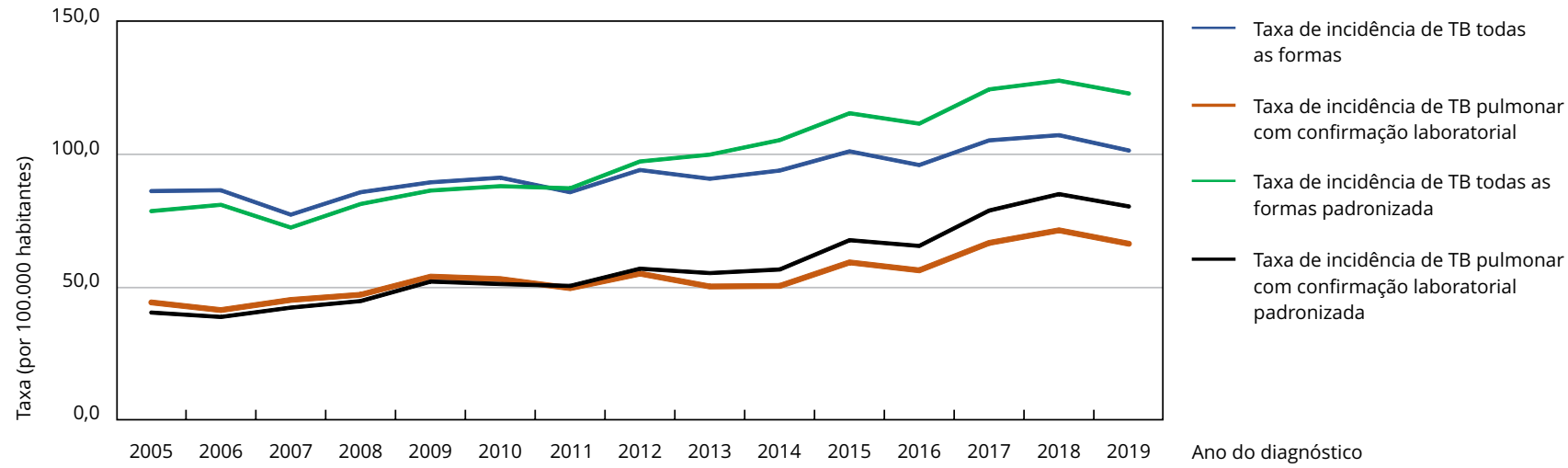

Fonte: Secretaria Municipal de Saúde de Manaus/Sistema de Informação de Agravos de Notificação-tuberculose (dados atualizados até 12 de janeiro de 2020).

A taxa bruta de mortalidade por TB no período de 2006 a 2019 apresenta aparente aumento. Ao analisar a taxa padronizada, verifica-se, visualmente, que a partir 2013 há um afastamento da taxa bruta, que se acentua da partir de 2017 (ano piloto da implantação do protocolo), dando ideia de aumento (Figura 4). Como a taxa de mortalidade não apresenta distribuição normal, a variação percentual foi utilizada para julgar esse aumento. Observa-se variação percentual positiva no período de 2006 a 2009 (22,2\%), com decréscimo entre 2010 e 2013 (2,9\%), voltando a aumentar de 2014 a 2016 (19,5\%), apresentando variação percentual mais acentuada de 2017 a 2019 (22,6\%).

Em relação à proporção de óbito por TB sem menção de confirmação laboratorial, houve redução na variação percentual de -3,9\% no período 2006-2016 (período pré-implantação do protocolo) e 64,6\% no período de 2017-2019 (período pós-implantação do protocolo), conforme a Figura 5.

\section{Discussão}

A vigilância do óbito com menção de TB é uma das estratégias inovadoras que compõem o Pilar 1 do Plano Brasileiro pelo Fim da TB, e busca fortalecer a prevenção e o cuidado integrado centrados na pessoa com TB 24. A abordagem metodológica utilizada nesse estudo constitui uma inovação e contribuiu com a identificação de potencialidades e fragilidades na implantação da vigilância do óbito de TB. Os resultados encontrados mostram que a implantação da vigilância do óbito com menção de TB em Manaus foi considerada parcialmente implantada, e as diferenças entre os seus componentes merecem o adequado entendimento, a fim identificar as fragilidades e fortalezas da implantação do mencionado no protocolo.

O modelo lógico de um estudo que avaliou a implantação da vigilância do óbito infantil 22, semelhante às recomendações nacionais para vigilância do óbito com menção de TB, serviu de base e mostrou-se pertinente a esta avaliação. Com o modelo voltado à vigilância do óbito por TB, foi possível fazer julgamento dos componentes de "identificação do óbito", de "investigação epidemiológica", da "discussão dos óbitos" e dos "desdobramentos de propostas à rede de assistência e qualificação dos sistemas de informação”. Desse modo, parte-se de uma proposta que se mostrou aplicável e adequada à vigilância do óbito por TB. 


\section{Figura 4}

Taxa bruta e padronizada da mortalidade de tuberculose (TB) todas as formas e pulmonares com confirmação laboratorial. Manaus, Amazonas, Brasil, 2006 a 2019.

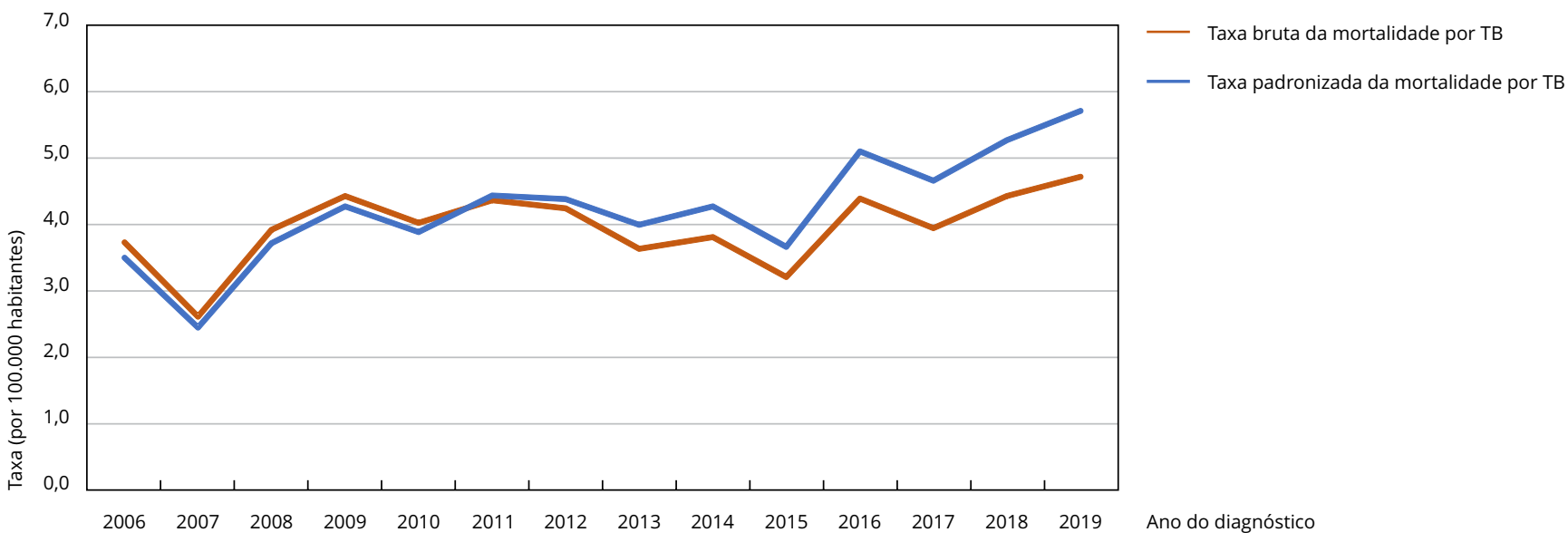

Fonte: Secretaria Municipal de Saúde de Manaus/Sistema de Informações sobre Mortalidade (dados atualizados até 28 de janeiro de 2020).

\section{Figura 5}

Proporção de óbito por tuberculose sem e com menção de confirmação laboratorial. Manaus, Amazonas, Brasil, 2006 a 2019.

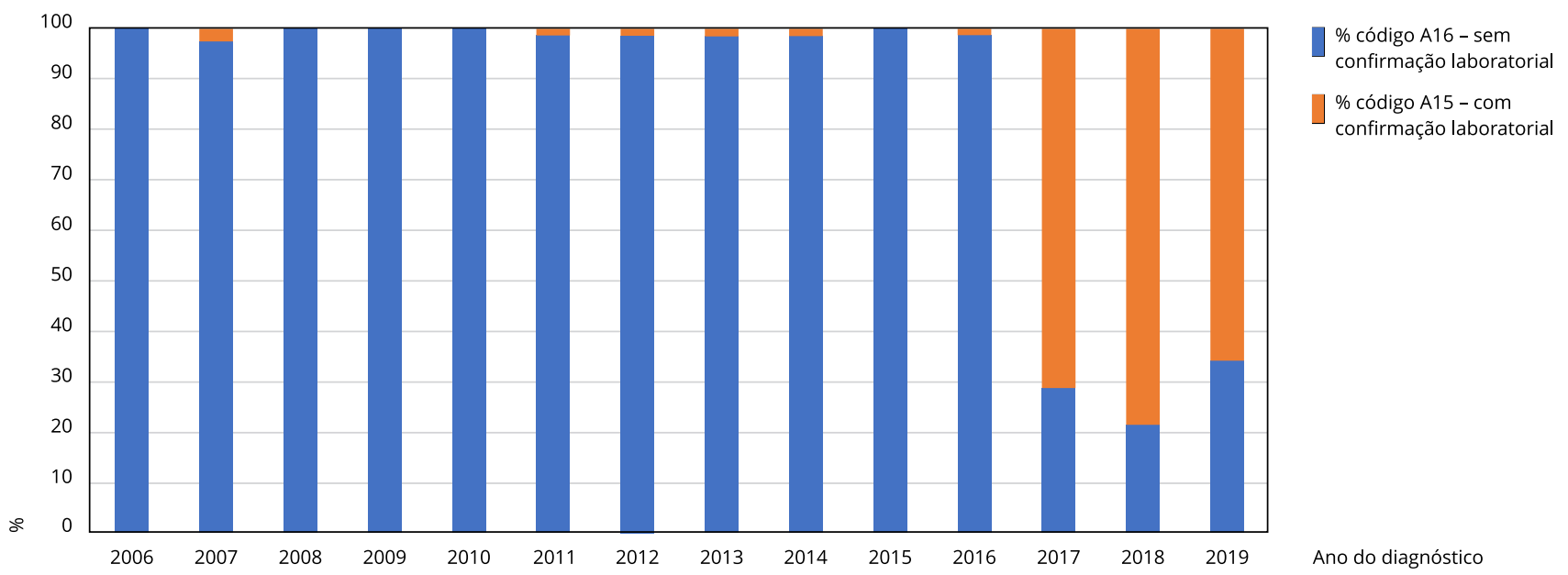

Fonte: Sistema de Informações sobre Mortalidade (dados atualizados até 28 de janeiro de 2020).

Na avaliação do componente "identificação do óbito", as atividades de alimentação dos dados no SIM e o envio dos registros de óbitos ao PCT de Manaus contribuíram para o elevado percentual identificado no resultado da abordagem do "processo". O fato de os responsáveis por essas atividades não terem capacitação/curso de atualização na vigilância do óbito com TB pode ser um fator limitante neste componente, o qual precisa ser superado. Em outros estudos que avaliaram a implantação do SIM, a ausência de capacitação dos recursos humanos, de supervisão e controle, e a alta rotatividade 
de técnicos para o gerenciamento da informação, foram encontrados em nível municipal como fatores que comprometeram a implantação do SIM na sua inteireza 25,26,27.

A "investigação do óbito" com menção de TB é outra etapa importante e direciona o trabalho da equipe do PCT municipal. Nesse sentido, foi atribuída maior pontuação ao componente, e seu resultado foi importante para definir o grau de implantação da intervenção. A abordagem "estrutura" parece não ser a principal dificuldade enfrentada pelo PCT de Manaus, observou-se uma estrutura mínima necessária para a investigação do óbito. Contudo a quantidade de profissionais da equipe do PCT e da vigilância epidemiológica municipal que realizam investigação, e o número de NVEH que enviam fichas de investigação do óbito ao PCT municipal foram pontos críticos encontradas que implicaram na abordagem do "processo". Estudo prévio que avaliou a implantação dos núcleos hospitalares de epidemiologia destaca que as atividades de vigilância epidemiológica desempenhadas pelos NVEH podem enfrentar entraves em relação à não contratação de recursos humanos, à falta de capacitação e à pouca viabilidade por parte dos gestores em relação ao papel dos núcleos 28 .

A realização das atividades de investigação do óbito, em conformidade com protocolos oficiais, também foi um desafio vivenciado com a implantação da vigilância do óbito materno. No Brasil, a proporção de óbitos maternos investigados era baixa, mas, a partir da implementação de políticas públicas voltadas ao fortalecimento dessa estratégia, a cobertura ampliou-se de 55\%, em 2009, para $80 \%$, em 2011 29. No âmbito da vigilância do óbito infantil, estudo realizado no Nordeste do Brasil apontou que $95,1 \%$ dos óbitos infantis tinham investigação 22, reforçando que os obstáculos ao longo dos anos vêm sendo superados com esforços dos três níveis do governo - o federal, o estadual e o municipal 30 . Da mesma maneira, espera-se que os componentes da vigilância do óbito com menção de TB sejam completamente implantados e as lacunas da operacionalização do protocolo sejam superadas.

As recomendações nacionais e internacionais para vigilância do óbito preconizam que, uma vez concluída a investigação, um grupo de trabalho seja formado para discussão dos determinantes da ocorrência do óbito 3,30,31. O fato de esse componente estar aquém do esperado compromete a vigilância do óbito do PCT de Manaus. Um fator que pode implicar no trabalho de um grupo de discussão é a escassez de profissionais do sistema de saúde ou mesmo a sobrecarga e diversas demandas, ficando pouco ou nenhum espaço na agenda de trabalho para a discussão de casos, e essa mesma percepção foi identificada no estudo que avaliou a vigilância do óbito infantil 22.

A etapa final da investigação do óbito com menção de TB são as recomendações elaboradas pelo grupo de trabalho ${ }^{3}$. Ao componente "encaminhamento de propostas e qualificação dos sistemas de informação" foi atribuído menor pontuação, por ser desdobramento das atividades anteriores. Apesar de esse componente ser classificado como não implantado, a avaliação de indicadores de resultado sugere que mudanças no processo de trabalho podem ter ocorrido, pois houve redução dos óbitos sem confirmação laboratorial para TB e redução dos óbitos com menção de TB na causa básica registrados no SIM e não notificados no SINAN, comparado ao panorama da mortalidade por TB publicado pelo PNCT 4. Entretanto o controle e resgate das alterações realizadas no SIM pode ter sofrido prejuízos pela falta de automatização ou de instrumento de registro, dificultando mensurá-las.

A implementação nas ações de vigilância de óbito com menção de TB pode ter impacto nos indicadores de incidência e mortalidade por TB 5,7,9. Um sistema de vigilância e de resposta aos óbitos por TB bem sucedido seria um grande passo no sentido de melhorar a mensuração da carga de mortalidade e mesmo de incidência de TB no país 32 . Este estudo corrobora a literatura citada, pois após a implantação da vigilância do óbito em Manaus houve acentuação na curva da taxa de incidência de TB (todas as formas e com confirmação laboratorial) e da taxa de mortalidade pela doença, sugerindo que a realização das atividades de vigilância do óbito, ainda que parcialmente implantada, como mostra o resultado deste estudo, pode ter trazido à luz a situação da morbimortalidade por TB em Manaus.

Sabe-se que os resultados do impacto desse tipo de implantação dificilmente aparecem em curto prazo, entretanto esse aumento dos registros pode gerar algumas hipóteses, se ocorreu pela qualificação dos sistemas de informação disponíveis, com diminuição da subnotificação ou se houve também aumento real dos casos de TB.

Espera-se que, em longo prazo, as ações de vigilância do óbito de TB contribuam para identificar fatores associados ao óbito, melhorando o cuidado aos pacientes de TB e reduzindo o número de mortes pela doença. 
O cenário epidemiológico é desafiador para a equipe do PCT de Manaus. Enquanto o Brasil e as cinco regiões apresentam comportamento temporal decrescente estatisticamente significativo da mortalidade por TB 1,33,34, a incidência de TB todas as formas e pulmonares com confirmação laboratorial em Manaus apresentam tendência de aumento e a mortalidade pela doença permanece muito acima das metas estimadas para eliminação da TB como problema de saúde pública 1 .

Contudo a implantação parcial do protocolo de vigilância do óbito com menção de TB em Manaus indica que os esforços do PCT municipal são bem-sucedidos. Embora seja necessário superar os desafios para o adequado funcionamento da vigilância do óbito, é necessário que os profissionais que compõem a equipe do PCT municipal e vigilância epidemiológica municipal estejam envolvidos em todas as etapas que permeiam a vigilância do óbito com menção de TB, e que fortaleça a parceria entre o PCT municipal e estadual, a fim de mobilizar e sensibilizar os profissionais dos NVEH para essa estratégia. É essencial que todos os atores estejam capacitados para as atividades de vigilância do óbito de TB, incluindo os técnicos que atuam no âmbito do SIM. Acredita-se que a organização do processo do trabalho seja fundamental para execução das investigações e discussões dos óbitos. Sugere-se ainda que, em nível nacional, seja elaborada uma ferramenta para monitoramento da vigilância do óbito, permitindo assim a construção de banco de dados com as informações necessárias para cálculo de indicadores de monitoramento da vigilância do óbito com menção de TB.

Entre as limitações deste trabalho, destaca-se a ausência de modelo lógico e matriz de julgamento prévios e validados para esse tipo de avaliação, assim como a ausência de estudos avaliativos de implantação da vigilância do óbito por TB no Brasil, o que implica na imprecisão de rotinas de serviços de diferentes contextos e alguns parâmetros a serem adotados. Entretanto este modelo permitiu avaliar a implantação da vigilância do óbito de TB e a ausência de validação prévia não comprometeu os resultados e sua interpretação. Apesar disso, é importante realizar outros estudos para validação do modelo aqui proposto em novas pesquisas, para melhor adequação do modelo lógico, dos critérios e indicadores considerados. A ausência de um banco de dados com informações dos óbitos investigados, para cálculo dos indicadores de monitoramento da vigilância do óbito com menção de TB, foi outra limitação considerada neste estudo. Contudo não trouxe prejuízos nos resultados, uma vez que, no questionário respondido pelo coordenador municipal da TB, foram contemplados tais indicadores, incluindo os de resultados, obtidos a partir de uma ferramenta desenvolvida por um estatístico do Departamento de Vigilância Epidemiológica e Ambiental da Semsa-Manaus, no programa Access (https://www.microsoft.com/en-us/microsoft-365/access), que pareia os bancos de dados SIM e SINAN para verificação de tais inconsistências. Por fim, o uso dos dados secundários obtidos a partir dos sistemas de informação traz por si a possibilidade de falhas, entre elas o preenchimento errôneo dos campos, o que pode afetar na qualidade dos dados registrados e na análise de interesse, mas que possuem vantagens pela cobertura populacional 35 .

\section{Conclusão}

A implantação do protocolo de vigilância do óbito com menção de TB foi classificada como parcialmente implantada em Manaus, e isso indica que há necessidade de aperfeiçoamento das ações quanto à qualificação dos sistemas de informação, SIM e SINAN, da vigilância laboratorial para diagnóstico dos casos e discussão dos óbitos com encaminhamento de propostas para melhorar o acompanhamento dos casos de TB.

O impacto da implantação do protocolo de vigilância do óbito com menção de TB é evidenciado pela evolução dos indicadores de incidência e mortalidade por TB, assim como os óbitos com confirmação laboratorial, os quais apontam que as atividades de vigilância estão sendo realizadas, ainda que parcialmente. Observou-se também que mudanças no processo de trabalho aconteceram na equipe do SIM e do PCT.

Ressalta-se que os componentes essenciais da vigilância do óbito avaliados neste estudo precisam ser implementados para que realmente se conheçam nós críticos que permeiam o PCT de Manaus. 


\section{Colaboradores}

D. C. Cordeiro contribuiu na elaboração do projeto de pesquisa, e na redação do artigo. M. J. F. Gonçalves contribuiu na redação do artigo. As autoras aprovaram a versão final para publicação.

\section{Informações adicionais}

ORCID: Dinah Carvalho Cordeiro (0000-00031830-8532); Maria Jacirema Ferreira Gonçalves (0000-0002-8460-8501).

\section{Agradecimentos}

À Secretaria Municipal de Saúde de Manaus pela colaboração e apoio. À banca de defesa da dissertação: professora Dra. Anete Trajman (UFRJ) e professora Dra. Laura Maria Vidal Nogueira (UEPA), pelas contribuições, e a Coordenação de Aperfeiçoamento de Pessoal de Nível Superior (CAPES Código de financiamento 001).

\section{Referências}

1. Secretaria de Vigilância em Saúde, Ministério da Saúde. Tuberculose 2020. Boletim Epidemiológico de Tuberculose 2020; número especial.

2. Castelo A, Mathiasi PA, Iunes R, Kritski AL, Dalcolmo M, Fiuza de Melo F, et al. Cost effectiveness of antituberculosis interventions. PharmacoEconomics 1995; 8:385-99.

3. Departamento de Vigilância das Doenças Transmissíveis, Secretaria de Vigilância em Saúde, Ministério da Saúde. Protocolo para vigilância do óbito com menção de tuberculose nas causas de morte. Brasília: Ministério da Saúde; 2017.

4. Departamento de Vigilância das Doenças Transmissíveis, Secretaria de Vigilância em Saúde, Ministério da Saúde. Panorama da tuberculose no Brasil: a mortalidade em números. Brasília: Ministério da Saúde; 2016.

5. Romero ROG, Ribeiro CMC, Sá LD, Villa TCS, Nogueira JDA. Subnotificação de casos de tuberculose a partir da vigilância do óbito. Rev Eletrônica Enferm 2016; 8:e1161.

6. Departamento de Vigilância das Doenças Transmissíveis, Secretaria de Vigilância em Saúde, Ministério da Saúde. Manual de recomendações para o controle da tuberculose no Brasil. Brasília: Ministério da Saúde; 2019.

7. Peres DA, Façanha MC, Viana Júnior AB. Incremento de casos e melhoria da informação sobre tuberculose no Estado do Ceará, Brasil, após o relacionamento de bases de dados. Cad Saúde Colet (Rio J.) 2017; 25:491-7.

8. Bartholomay P, Oliveira GP, Pinheiro RS, Vasconcelos AMN. Melhoria da qualidade das informações sobre tuberculose a partir do relacionamento entre bases de dados. Cad Saúde Pública 2014; 30:2459-69.

9. Selig L, Kritski AL, Cascão AM, Braga JU, Trajman A, Carvalho RMG. Proposta de vigilância de óbitos por tuberculose em sistemas de informação. Rev Saúde Pública 2010; 44:1072-8.

10. Hartz ZMA, organizadora. Avaliação em saúde: dos modelos conceituais à prática na análise da implantação de programas. Rio de Janeiro: Editora Fiocruz; 1997.

11. Donabedian A. Explorations in quality assessment: the definitions of quality and approaches to its assessment. v. 1. Ann Arbor: ACHE Management Series; 1980.

12. Tanaka OY, Ribeiro EL, Almeida CAL. Avaliação em saúde: contribuições para incorporação no cotidiano. Rio de Janeiro: Editora Atheneu; 2017.

13. Adamu AL, Gadanya MA, Abubakar IS, Jibo AM, Bello MM, Gajida AU, et al. High mortality among tuberculosis patients on treatment in Nigeria: a retrospective cohort study. BMC Infect Dis 2017; 17:170.

14. Blount RJ, Pascopella L, Catanzaro DG, Barry PM, English PB, Segal MR, et al. Traffic-related air pollution and all-cause mortality during tuberculosis treatment in California. Environ Health Perspect 2017; 125:097026. 
15. Queiroz AAR, Berra TZ, Garcia MCC, Barry PM, English PB, Segal MR, et al. Spatial pattern and temporal trend of mortality due to tuberculosis. Rev Latinoam Enferm 2018; 26:e2992.

16. Yamamura M, Santos-Neto M, Santos RAN, Garcia MCC, Nogueira JA, Arcêncio RA. Epidemiological characteristics of cases of death from tuberculosis and vulnerable territories. Rev Latinoam Enferm 2015; 23:910-8.

17. Bartholomay P, Oliveira GP, Pinheiro RS, Vasconcelos AMN. Melhoria da qualidade das informações sobre tuberculose a partir do relacionamento entre bases de dados. Cad Saúde Pública 2014; 30:2459-70.

18. Instituto Brasileiro de Geografia e Estatística. Manaus (AM). https://www.ibge.gov.br/cida des-e-estados/am/manaus.html (acessado em $11 /$ Set/2020).

19. e-Gestor Atenção Básica. Cobertura da atenção básica. https://egestorab.saude.gov.br/ paginas/acessoPublico/relatorios/relHisto ricoCoberturaAB.xhtml (acessado em 23/ Fev/2021)

20. Secretaria Municipal de Saúde de Manaus. Plano municipal de saúde de Manaus, 20182021. https://semsa.manaus.am.gov.br/wpcontent/uploads/2019/04/Plano-Municipalde-Sa\%C3\%BAde-de-Manaus-2018-2021.pdf (acessado em 14/Dez/2020).

21. Marreiro LS, Cruz MA, Oliveira MNF, Garrido MS. Tuberculose em Manaus, Estado do Amazonas: descentralização. Epidemiol Serv Saúde 2009; 18:237-42.

22. Oliveira CM, Frias PG, Bonfim CV, Antonino VCS, Nascimento JDT, Medeiros ZM. Assessment of infant mortality surveillance: case study. Rev Bras Saúde Mater Infant 2017; 17:801-15.

23. Heufemann NEC, Gonçalves MJF, Garnelo ML. Avaliação do programa de controle da tuberculose em cenário Amazônico: desafios em Coari. Acta Amaz 2013; 43:33-42.

24. Secretaria de Vigilância em Saúde, Ministério da Saúde. Brasil livre da tuberculose: plano nacional pelo fim da tuberculose como problema de saúde pública. Brasília: Ministério da Saúde; 2017.

25. Figueirôa BQ, Frias PG, Vanderlei LCM, Vidal SA, Carvalho PI, Pereira CCB, et al. Avaliação da implantação do Sistema de Informações sobre Mortalidade no estado de Pernambuco em 2012. Epidemiol Serv Saúde 2019; 28:e2018384.
26. Frias PG, Pereira PMH, Andrade CLT, Szwarcwald CL. Sistema de Informações sobre Mortalidade: estudo de caso em municípios com precariedade dos dados. Cad Saúde Pública 2008; 24:2257-66.

27. Pereira CCB, Vidal SA, Carvalho PI, Frias PG. Avaliação da implantação do Sistema de Informações sobre Nascidos Vivos (Sinasc) em Pernambuco. Rev Bras Saúde Mater Infant 2013; 13:39-49.

28. Mendes MFM. Avaliação da implantação dos núcleos de epidemiologia em hospitais de alta complexidade da rede pública de saúde no Recife-PE [Dissertação de Mestrado]. Recife: Instituto Aggeu Magalhães, Fundação Oswaldo Cruz; 2001.

29. Seminário sobre a vigilância do óbito fetal, infantil e materno no brasil. Dourados Agora 2013; 10 abr. https://www.douradosagora. com.br/2013/04/10/seminario-sobre-a-vigi lancia-do-obito-fetal-infantil-e-materno-no -brasil/ (acessado em 14/Out//2020).

30. Departamento de Análise de Situação de Saúde, Secretaria de Vigilância em Saúde, Ministério da Saúde. Guia de vigilância epidemiológica do óbito materno. Brasília; Ministério da Saúde; 2009.(Série A. Normas e Manuais Técnicos).

31. Asha D. Community based child death review. http://www.nrhmhp.gov.in/sites/default/ files/files/Child\%20death\%20Review\%20 guidelines.pdf (acessado em 21/Jan/2022).

32. Bierrenbach AL, Selig L. O Brasil precisa de um sistema nacional de vigilância de óbito por tuberculose? Cad Saúde Pública 2015; 31:661-2.

33. Souza CDF, Paiva JPS, Silva LF, Leal TC, Magalhães MAFM. Trends in tuberculosis mortality in Brazil (1990-2015): joinpoint analysis. J Bras Pneumol 2019; 45:e20180393.

34. Oliveira A, Alfonso A, Miranda R, Oliveira TRS. Perfil epidemiológico da tuberculose no Nordeste do Brasil: série temporal de 2008 a 2018. Research, Society and Development 2020; 9:108922129.

35. Coeli CM. Sistemas de Informação em Saúde e uso de dados secundários na pesquisa e avaliação em saúde. Cad Saúde Colet (Rio J.) 2010; 18:335-6. 


\section{Abstract}

The study aimed to assess the degree of implementation and impact of the protocol for surveillance of deaths from tuberculosis $(T B)$ in a large city with a high TB burden. For this evaluative study, a log frame model with a assessment matrix was elaborated. Primary data were collected with a questionnaire applied to healthcare workers, and secondary data were obtained from the Manaus Municipal Health Department, Amazonas State, Brazil. The impact of the protocol's adoption was analyzed according to the indicators of results and evolution of TB incidence and mortality rates based on simple linear regression and percent change. The degree of implementation of surveillance of deaths with mention of $T B$ was partial (51\%). Evolution of TB incidence and mortality showed a significant upward trend in TB incidence from 2006 to 2019. TB mortality and the proportion of deaths with laboratory confirmation of $T B$ showed an increase in the protocol's implementation, indicating an increase in surveillance. Partial implementation of TB mortality surveillance suggests the need for changes in the work processes of the team managing the Brazilian Mortality Information System and the Municipal TB Control Program. The changes demonstrate improvement of information on $T B$ deaths and the contribution of surveillance of $T B$ diagnosis to definition of cases with TB laboratory confirmation.

Tuberculosis; Mortality; Health Evaluation; Epidemiologic Surveillance

\section{Resumen}

El objetivo de este estudio fue evaluar el grado de implantación y de impacto del Protocolo de vigilancia de óbitos por tuberculosis (TB) en un municipio de gran tamaño y con elevada carga de TB. Para esta investigación evaluativa se elaboró un modelo lógico, así como una matriz de juicio. Los datos primarios se recogieron a partir de un cuestionario aplicado a profesionales y los datos secundarios se obtuvieron de la Secretaría Municipal de Salud de Manaos, Amazonas, Brasil. El impacto de la adopción del protocolo se analizó mediante indicadores de resultados, así como la evolución de las tasas de incidencia y mortalidad por TB, a partir de una regresión lineal simple y variación porcentual. El grado de implantación de la vigilancia del óbito con mención de TB identificado fue parcial (51\%). En cuanto a la evolución de los indicadores de incidencia y mortalidad por TB, se observó una tendencia significativa de aumento de la tasa de la incidencia de TB en el período de 2006 a 2019. La mortalidad por TB, así como la proporción de los óbitos con confirmación de laboratorio para $T B$ presentaron un incremento tras la implantación del protocolo, indicando un aumento de la vigilancia. La implantación parcial de la vigilancia de óbitos por TB indica que son necesarios cambios en el proceso de trabajo del equipo que gestiona el Sistema de Informaciones sobre Mortalidad y el Programa de Control de la TB municipal. Los cambios observados demuestran más cualificación respecto a la información del óbito por $T B$, así como la conclusión de que vigilancia en el diagnóstico de la TB contribuyó a la definición de casos con confirmación de TB por parte de laboratorios.

Tuberculosis; Mortalidad; Evaluación en Salud; Vigilancia Epidemiológica
Recebido em 30/Dez/2020

Versão final reapresentada em 29/Mai/2021

Aprovado em 20/Ago/2021 